\title{
Halo-gravity traction for the treatment of pediatric cervical spine disorders
}

\author{
Bram P. Verhofste, MD, 1,2 Michael P. Glotzbecker, MD, ${ }^{3,4}$ Craig M. Birch, MD, 1,2 Nora P. O’Neill, BA,,2 \\ and Daniel J. Hedequist, MD1,2
}

\begin{abstract}
${ }^{1}$ Department of Orthopaedic Surgery, Boston Children's Hospital; ${ }^{2}$ Harvard Medical School, Boston, Massachusetts; ${ }^{3}$ Department of Orthopaedic Surgery, University Hospitals Cleveland Medical Center; and ${ }^{4}$ Department of Orthopaedic Surgery, Rainbow Babies and Children's Hospital, Cleveland, Ohio
\end{abstract}

\begin{abstract}
OBJECTIVE Halo-gravity traction (HGT) is an effective and safe method for gradual correction of severe cervical deformities in adults. However, the literature is limited on the use of HGT for cervical spine deformities that develop in children. The objective of the present study was to evaluate the safety and efficacy of HGT for pediatric cervical spine deformities.

METHODS Twenty-eight patients (18 females) whose mean age was $11.3 \pm 5.58$ years (range 2-24.9 years) underwent HGT. Common indications included kyphosis $(n=12)$, rotatory subluxation $(n=7)$, and basilar invagination $(n=6)$. Three children $(11 \%)$ received traction to treat severe occipitocervical instability. For these 3 patients, traction combined with a halo vest, with bars attached rigidly to the vest, but with the ability to slide through the connections to the halo crown, was used to guide the corrective forces and moments in a specific and controlled manner. Patients ambulated with a wheelchair or halo walker under constant traction. Imaging was done before and during traction to evaluate traction efficacy. The modified Clavien-Dindo-Sink classification was used to categorize complications.
\end{abstract}

RESULTS The mean duration of HGT was 25 days (IQR $13-29$ days), and the mean traction was $29 \% \pm 13.0 \%$ of body weight (IQR $19 \%-40 \%$ of body weight). The mean kyphosis improved from $91^{\circ} \pm 20.7^{\circ}$ (range $64^{\circ}-122^{\circ}$ ) to $56^{\circ} \pm 17.6^{\circ}$ (range $32^{\circ}-96^{\circ}$ ) during traction and corresponded to a mean percentage kyphosis correction of $38 \% \pm 13.8 \%$ (range $21 \%-57 \%$ ). Twenty-five patients (89\%) underwent surgical stabilization, and 3 patients (11\%) had rotatory subluxation that was adequately reduced by traction and were treated with a halo vest as their definitive treatment. The mean hospital stay was 35 days (IQR 17-43 days).

Nine complications (32\%) occurred: 8 grade I complications (28\%), including 4 cases of superficial pin-site infection (14\%) and 4 cases of transient paresthesia (14\%). One grade II complication (4\%) was seen in a child with Down syndrome and a preexisting neurological deficit; this patient developed flaccid paralysis that rapidly resolved with weight removal. Six cases (21\%) of temporary neck discomfort occurred as a sequela of a preexisting condition and resolved without treatment within 24-48 hours.

CONCLUSIONS HGT in children is safe and effective for the gradual correction of cervical kyphosis, atlantoaxial subluxation, basilar invagination, and os odontoideum. Cervical traction is an additional tool for the pediatric spine surgeon if uncertainties exist that the spinal alignment required for internal fixation and deformity correction can be safely achieved surgically. Common complications included grade I complications such as superficial pin-site infections and transient paresthesias. Halo vest gravity traction may be warranted in patients with baseline neurological deficits and severe occipitocervical instability to reduce the chance of catastrophic movement.

https://thejns.org/doi/abs/10.3171/2019.10.PEDS19513

KEYWORDS pediatric; children; cervical spine; halo-gravity traction; halo vest; efficacy; complications; adverse events; modified Clavien-Dindo-Sink classification

$\mathrm{S}$ TAGNARA popularized halo-gravity traction (HGT) in 1971 as a method to use the natural force of gravity to counteract pathological forces causing severe thoracic scoliosis, kyphosis, or kyphoscoliosis. ${ }^{26,27}$ Traction causes soft-tissue relaxation that gradually improves spi- nal alignment in an attempt to reduce the amount of force or surgical exposure required for the stabilization of severe spinal deformities and to decrease the risk of perioperative neurological deficits. ${ }^{1,3-5,15,22,25,27,30}$ A halo crown with 4-8 pins is placed in the operating room and stepwise distrac-

ABBREVIATIONS AE = adverse event; ARS = atlantoaxial rotatory subluxation; BW = body weight; HGT = halo-gravity traction; HVGT = halo-vest-gravity traction; IONM = intraoperative neuromonitoring; LOS = length of stay; NF1 = neurofibromatosis type 1; SAC = space available for the spinal cord; SCC = spinal cord compression. SUBMITTED September 4, 2019. ACCEPTED October 28, 2019.

INCLUDE WHEN CITING Published online December 27, 2019; DOI: 10.3171/2019.10.PEDS19513. 
tion is performed until $30 \%-50 \%$ of body weight (BW) is reached. $3,15,19,22,30$ Weight in this range is usually tolerated well, and immediate mobilization is allowed with a wheelchair or halo walker. ${ }^{3,15}$

Daily pin-site care and neurological evaluation are required and can prevent most traction-related complications. Mild complications such as cervical discomfort, pin-site infections, and pin loosening are common and are easily managed with muscle relaxants, antibiotics, and pin tightening. ${ }^{3,9,19,21,22,30}$ More severe complications such as cranial nerve dysfunction, subdural abscesses, vertebral artery aneurysms, and paraplegia are also rarely reported. $4,9,10,14,31$

Good outcomes of preoperative HGT are reported in adults with severe basilar invagination, cervical kyphosis, or irreducible atlantoaxial subluxation in which singlestage realignment or instrumentation may be problematic. ${ }^{13,17,20,24,25,32}$ Cervical traction reduces the difficulty of anterior surgery in severely kyphotic patients and alleviates the need for transoral decompression in cases of complex craniocervical junction malformations. The literature on HGT for the management of cervical spine disorders that develop in children is limited to small case series that are often outdated, include adults, or utilize traction intra- and postoperatively. $1,10,13,14,16,18,24,28,31$ To our knowledge, this is the largest series dedicated to evaluating the efficacy and safety of HGT for pediatric cervical spine disorders.

The goals of this retrospective case series were as follows: 1) to describe the benefits and efficacy of HGT for the treatment of cervical spine disorders that develop in children; 2) to review the safety and complications using the modified Clavien-Dindo-Sink classification; and 3) to provide a protocol for HGT with recommendations from an experienced tertiary pediatric referral center.

\section{Methods}

A retrospective consecutive examination of children who underwent HGT during a 15-year period (2003-2018) was performed after IRB approval. All patients were treated at a single pediatric tertiary referral center (Boston Children's Hospital). Patients $<25$ years of age diagnosed with occipitocervical or cervical instability, cervical kyphosis, basilar invagination, atlantoaxial rotatory subluxation (ARS), os odontoideum, or cervical arthritis with deformity were included. Due to their complex medical histories, these patients are often medically active until 25 years of age at pediatric tertiary referral institutions. Exclusion criteria were head halter traction, predominant thoracic deformities, cervicothoracic disorders with $\mathrm{C} 7$ as the highest affected level, inadequate HGT data, or traction initiated at an outside institution. The objective was to evaluate the safety and efficacy of perioperative HGT, and cases with $<1$ year of follow-up data were included.

\section{Data Collection}

Patient demographics, HGT characteristics, and surgical data were collected using the electronic medical records system. Fusion was evaluated when sufficient postoperative imaging studies were available. Fusion could not be assessed in 13 patients because a high number of inter- national patients are treated at our institution. These cases were included in the analysis, however, to accurately represent the cervical spine population treated at the pediatric tertiary referral centers that perform HGT. These patients had complete records of their in-hospital HGT admission, which was the objective of the study.

\section{Radiographic Parameters}

Serial radiographs were used to evaluate kyphosis before HGT, during traction, postoperatively, and at the latest follow-up. Absolute kyphosis correction was calculated as follows: [pre-HGT - in-traction], and \% kyphosis correction as: [(absolute correction/pre-HGT) $\times 100 \%$ ]. A method was developed to maintain traction during CT scanning. The severity of basilar invagination was assessed on serial CT scans using McRae's line, defined as a line connecting the inner foramen magnum (basion to opisthion). ${ }^{6}$ The distance from the tip of the odontoid process perpendicular to this line was evaluated before and during HGT. Similarly, the space available for the spinal cord (SAC) was used to measure the effect of traction in cases of os odontoideum.

\section{Complication Data}

All HGT-related adverse events (AEs) were recorded using the modified Clavien-Dindo-Sink classification (grades I-V) for orthopedic complications. ${ }^{7}$ Superficial pin-site infections or transient paresthesias were classified as grade I or as complications that did require a deviation from routine care, had minimal clinical relevance, and were adequately treated with observation or minimal intervention (oral antibiotics, analgesics, or antipyretics). Grade II HGT-related complications included transient cranial nerve dysfunction, nerve palsies, or other temporary neurological deficits that required a deviation from HGT protocol (increased inpatient visits during hospitalization, close neurological monitoring, or weight removal). Grades I and II are considered minor complications.

Grade III-V complications are serious AEs classified as major complications. Subdural abscesses, cellulitis, or deep dermal infections were grade III complications that required surgical, interventional radiology, or endoscopic interventions, such as drainage, debridement, and intravenous antibiotics. Grade IV complications were life-threatening neurovascular AEs (cranial hemorrhage, spinal cord transection, vertebral artery injuries, or aneurysms) that necessitated intensive care unit admission and are divided into grade IVa (recovery with minimal loss of function) and IVb (long-term disability). Grade V indicates death. Temporary neck discomfort and pain were also recorded. These were considered sequelae of the preexisting cervical spine disorder and resolved within 24-48 hours without intervention.

\section{Patient Selection}

Factors influencing patient selection are as follows: etiology, failed conservative therapy, severity of spinal deformity, presence of neurological symptoms, and cases at high risk of neurological injury during single-stage surgical reduction. HGT can be used as adjuvant thera- 
py prior to surgery in severe cases of cervical kyphosis, basilar invagination, ARS, and os odontoideum. Traction is generally initiated for cervical kyphosis $>60^{\circ}$ in order to partially correct the deformity, reduce the amount of intraoperative manipulation required to obtain the desired correction, diminish the difficulty of anterior surgery in severely kyphotic patients, and minimize neurological deficits. Gradual improvement of the deformity also allows for increased biomechanical stability of rigid internal fixation constructs due to better anatomical alignment of the spine.

In patients with complex craniocervical junction malformations such as basilar invagination involving severe compression of the neural structures, we believe that traction is beneficial and prevents the need for transoral decompression. Partial preoperative correction, in patients with brainstem or spinal cord compression, improves neurological function, decreases surgical difficulties, reduces the likelihood of an invasive odontoid resection, and supports bony fusion after posterior-only procedures.

Cases of ARS that are severe, irreducible, chronic, or failed conservative therapy are recommended HGT to improve the orientation of $\mathrm{C} 1$ on $\mathrm{C} 2$ and improve head positioning. Presurgical reduction also increases the likelihood of successful $\mathrm{C} 1$ and $\mathrm{C} 2$ screw placement. In contrast to other cervical spine disorders, some patients with ARS in whom reduction is brought about by traction may not require surgical fixation and can be managed with conservative therapy after successful closed reduction.

Given the heterogeneity of patients and the severity of their disorders, it is difficult to determine objectively when traction achieves a result greater than what would have been achieved without presurgical HGT.

\section{Traction Protocol}

After induction of anesthesia, a halo crown was placed with 4-8 pins depending on bone density, patient size, and intended weight. Pin sites were prepared with chlorhexidine or alcohol without routine use of prophylactic antibiotics. Pins were placed in standard orientation and torqued to 4- to 8-inch pounds. A solution of local anesthetic and epinephrine was infiltrated around the pin sites to reduce postoperative pain. Halo-vest-gravity traction (HVGT) was used in 3 cases of severe occipitocervical instability when increased mechanical support was required to reduce the chance of catastrophic movement that can occur with bending and twisting motion. Traction in combination with a halo vest, with bars attached rigidly to the vest, but with the ability to slide through the connections to the halo crown, was used to guide the corrective forces and moments in a specific and controlled manner. Radiographs were acquired to ensure optimal position and spinal alignment following halo crown placement.

The ideal duration of traction is variable and depends on etiology, deformity flexibility, and goal traction weight. Patients typically receive traction for 3-8 weeks at 30\%$50 \%$ of their BW. Traction weight was based on BW $(\mathrm{kg})$ and etiology, represented as \% BW: ([traction weight/BW] $\times 100 \%)$. One to two pounds $(0.45-0.91 \mathrm{~kg})$ of weight was added daily until goal \%BW was achieved. Daily pin-site care was performed to prevent pin-site infections. Serial neurological examinations were performed to evaluate neurological status over time and to anticipate a deterioration in neurological function. Patients received continuous traction for 23-24 hours/day, and weight was only reduced when the patient received nursing care, was showering, experienced moderate to severe neck discomfort, or developed new neurological symptoms. Ambulation and activities of daily living were encouraged with the traction system attached to a wheelchair or halo walker. No activity restrictions were required.

\section{Surgical Technique}

Intraoperative neuromonitoring (IONM) consisting of somatosensory evoked potentials and motor evoked potentials was done in all cases. A combined anterior-posterior spinal fusion was performed in cases of severe cervical kyphosis to maintain the correction obtained during HGT, to maximize correctability through anterior soft-tissue releases, and to promote fusion with anterior discectomies combined with bone grafting. Internal fixation of basilar invagination, os odontoideum, and ARS was performed via a posterior-only approach. As a general rule, the patients were positioned in their halo with 5-10 pounds of traction unless the alignment was not adequate. In those cases, traction weight was not increased until stable potentials on IONM were achieved. It is the senior authors' experience that, with preoperative relaxation of structures due to HGT and the intraoperative relaxation due to anesthesia, the amount of weight needed in the operating room is negligible compared to the total weight reached during traction.

\section{Postoperative Management}

Postoperative immobilization with a rigid cervical collar or halo vest is required to maintain the stability of the internal fixation and facilitate bony fusion. The optimal external fixation period depends on the strength of the construct achieved during surgery and varies from case to case, with most patients discontinuing the collar or halo vest after evidence of bony fusion is observed on radiographs or CT scans. Pediatric patients, due to either age or other medical conditions/cognitive impairment, may not be able to comply with postoperative restrictions. This makes postoperative halo use more common in pediatric patients than it is in adults despite excellent fixation.

\section{Statistical Analysis}

Demographics and HGT characteristics were summarized using SPSS version 25 (IBM Corp.). When appropriate, data are expressed as the mean \pm SD or the median (IQR) for continuous variables. Frequency and percentages are used to summarize categorical variables. The paired Student t-test and Wilcoxon signed-rank tests were used, as appropriate. All tests were two-sided and $\mathrm{p}<0.05$ was considered significant.

\section{Results}

\section{Patient Demographics}

Twenty-eight children (18 females), whose mean age 
TABLE 1. Summary of characteristics in 28 pediatric patients

\begin{tabular}{|c|c|}
\hline Characteristic & Value (\% or range/IQR) \\
\hline \multicolumn{2}{|l|}{ Patient demographics } \\
\hline Age at HGT, yrs & $11.3 \pm 5.58($ range $2.0-24.9)$ \\
\hline No. of females & $18(64 \%)$ \\
\hline Height, cm & $133 \pm 27.5$ (range $71-177)$ \\
\hline Weight, kg & $35.3 \pm 24.33$ (range 9.4-119) \\
\hline \multicolumn{2}{|l|}{ Diagnosis } \\
\hline Cervical kyphosis & $12(43 \%)$ \\
\hline Rotatory subluxation & $7(25 \%)$ \\
\hline Basilar invagination & $6(21 \%)$ \\
\hline Os odontoideum & $2(7 \%)$ \\
\hline Cervical arthritis w/ deformity & $1(4 \%)$ \\
\hline \multicolumn{2}{|l|}{ Halo details } \\
\hline HGT & $25(89 \%)$ \\
\hline HVGT & $3(11 \%)$ \\
\hline \multicolumn{2}{|l|}{ No. of pins } \\
\hline 4 & $14(50 \%)$ \\
\hline 6 & $5(18 \%)$ \\
\hline 8 & $9(32 \%)$ \\
\hline Duration, days & $25 \pm 22.6$ (IQR 13-29) \\
\hline HGT weight, $\mathrm{kg}$ & $8.1 \pm 2.92($ IQR $5.6-9.4)$ \\
\hline$\% \mathrm{BW}, \mathrm{kg}^{*}$ & $29 \% \pm 13.0($ IQR $19 \%-40 \%)$ \\
\hline \multicolumn{2}{|l|}{ Halo complications } \\
\hline Total & $9(32 \%)$ \\
\hline Grade I & $8(29 \%)$ \\
\hline Superficial pin-site infection & $4(14 \%)$ \\
\hline Transient paresthesias & $4(14 \%)$ \\
\hline Grade II & $1(4 \%)$ \\
\hline Flaccid paralysis & $1(4 \%)$ \\
\hline \multicolumn{2}{|l|}{ Transient symptoms $\dagger$} \\
\hline Discomfort or neck pain & $6(21 \%)$ \\
\hline \multicolumn{2}{|l|}{ Surgical intervention } \\
\hline No. w/ surgery after HGT & $25(89 \%)$ \\
\hline LOS, days & $35 \pm 29.6$ (IQR 17-43) \\
\hline Follow-up duration, yrs & $1.7 \pm 1.51(\mathrm{IQR} 0.4-3.0)$ \\
\hline Fusion at latest follow-up $\ddagger$ & $15(100 \%)$ \\
\hline
\end{tabular}

Values are presented as the mean \pm SD (range or IQR) or as the number (\%) of patients unless otherwise stated.

${ }^{*}$ Final HGT weight measured in percentage of BW: [(halo weight/BW) $\left.\times 100 \%\right]$.

$\dagger$ Temporary neck discomfort was considered a sequela of the preexisting cervical spine disorder and rapidly resolved within 24-48 hours without intervention.

$\ddagger$ Fifteen cases had sufficient follow-up to evaluate radiographic fusion.

was $11.3 \pm 5.58$ years (range $2-24.9$ years), were identified (Table 1). Deformities included cervical kyphosis $(n=12)$, ARS $(n=7)$, basilar invagination $(n=6)$, os odontoideum $(n=2)$, and cervical arthritis with deformity $(n=1)$. Neurofibromatosis was the most frequent etiology $(n=6)$.

\section{HGT Characteristics}

Twenty-five patients (89\%) received standard HGT traction through a halo crown (Table 1). Three patients (11\%) with severe occipitocervical instability and spinal cord compression (SCC) were treated with HVGT to increase stability and reduce the risk of neurological deterioration: one patient was a 12.9-year-old boy with an uncharacterized syndromic disorder, severe basilar invagination, and odontoid retroflexion; one was a 10.9-year-old girl with Down syndrome and severe os odontoideum resulting in SCC, brainstem compression, and myelopathy (SAC 7.8 $\mathrm{mm}$ ); and one patient was a 2-year-old girl with a SOX9 gene mutation disorder and $85^{\circ}$ cervical kyphosis. The mean duration of traction was $25 \pm 22.6$ days (range 2-113 days; IQR 13-29 days), and the mean final weight was $29 \% \pm 13.0 \%$ (range $7 \%-58 \%$; IQR $19 \%-40 \%$ ) of BW (Tables 1 and 2).

\section{Efficacy}

Twelve children (43\%) with cervical kyphosis presented with a mean kyphotic deformity of $91^{\circ} \pm 20.7^{\circ}$ (range $64^{\circ}-$ $122^{\circ}$ ) (Table 2). The mean absolute correction and percentage of in-traction correction were $35^{\circ} \pm 16.3^{\circ}$ (range $18^{\circ}-68^{\circ}$ ) and $38 \% \pm 13.8 \%$ (range $21 \%-57 \%$ ), respectively (case 1; Figs. 1-3). The evolution of basilar invagination was assessed in 4 cases with serial CT scans. At presentation, the tip of the odontoid process was a mean of 4.9 $\pm 2.08 \mathrm{~mm}$ (range 3.0-7.1 mm) above McRae's line and improved during traction to $0.3 \pm 3.41 \mathrm{~mm}$ (range -4.9 to $3.0 \mathrm{~mm}$ ) (case 2; Figs. 4-6). The mean pre- and in-traction $\mathrm{SAC}$ values in cases of os odontoideum were $5.9 \pm 0.85$ $\mathrm{mm}$ (range 5.3-6.5 mm) and $11.4 \pm 0.35 \mathrm{~mm}$ (range 11.1$11.6 \mathrm{~mm})$, respectively.

\section{Safety}

Nine patients (32\%) experienced HGT-related complications. All were minor AEs classified as grade I or grade II complications (Tables 1 and 2). Eight grade I complications (28\%) were seen that included 4 superficial pinsite infections (14\%) easily treated with pin-site care and antibiotics. The remaining 4 grade I complications (14\%) were transient sensory complaints in the upper extremities that were documented for 24-48 hours and resolved without intervention. Of these, 3 (75\%) were unilateral upper-extremity paresthesias that occurred at 13 pounds (5.9 $\mathrm{kg}), 20$ pounds $(9.1 \mathrm{~kg})$, and 27 pounds $(12.2 \mathrm{~kg})$, respectively. One patient experienced transient bilateral upperextremity paresthesias on day 1 with 5 pounds $(2.3 \mathrm{~kg})$. Six children (21\%) developed temporary neck discomfort that occurred after a minimum of 15 pounds $(6.8 \mathrm{~kg})$ and resolved without intervention.

A grade II complication (4\%) occurred in a myelopathic patient with Down syndrome, os odontoideum, and severe SCC. The initial treatment consisted of HVGT to improve mechanical stability. The patient developed transient leftsided flaccid paralysis after the weight was increased from 5 to 10 pounds ( 2.3 to $4.5 \mathrm{~kg}$ ) on day 1 . The weakness resolved on weight removal, and HVGT resumed uneventfully on day 3. No patients experienced a major grade III$\mathrm{V}$ complication.

\section{Surgical Intervention}

Twenty-five patients (89\%) underwent spinal fusion, 


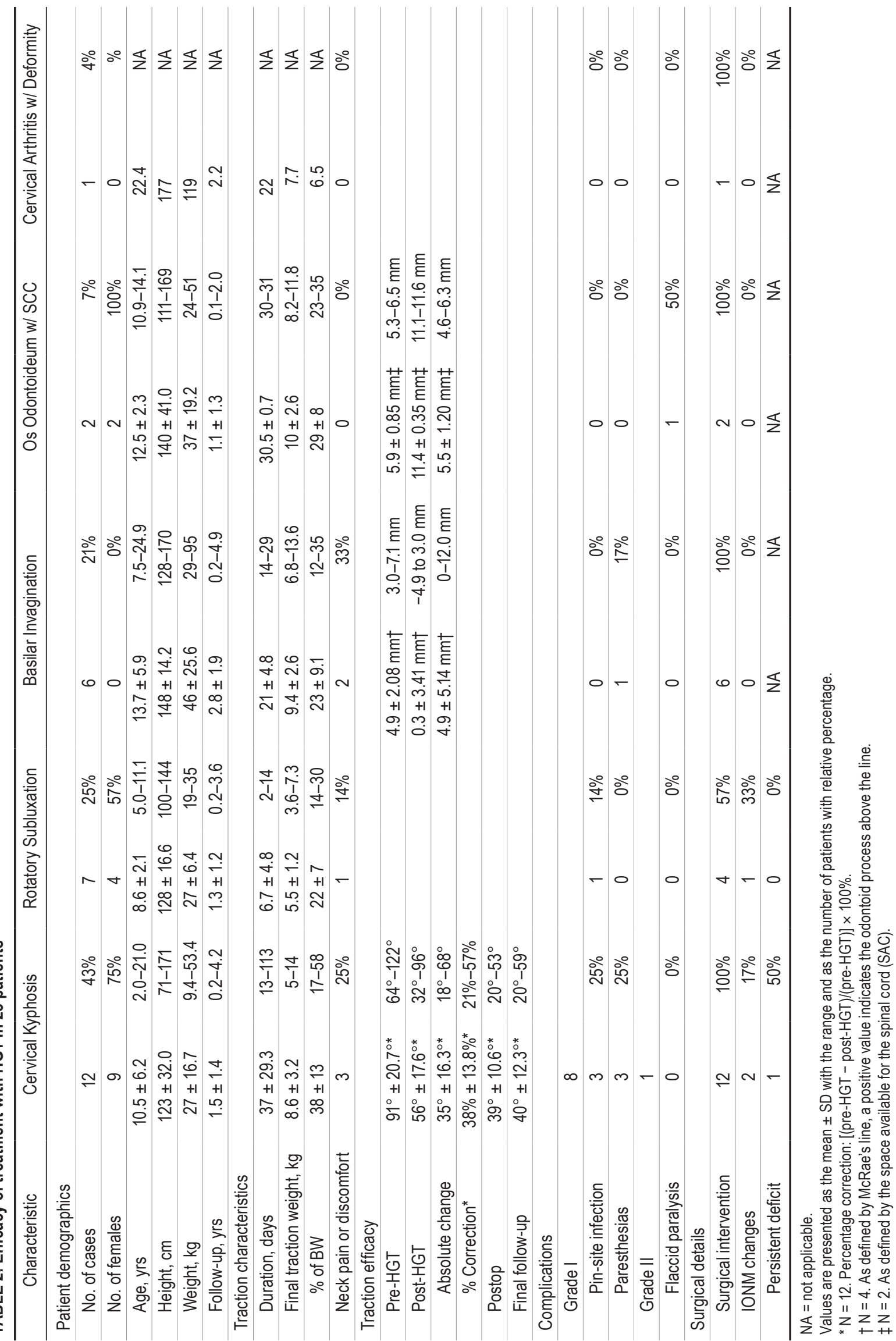




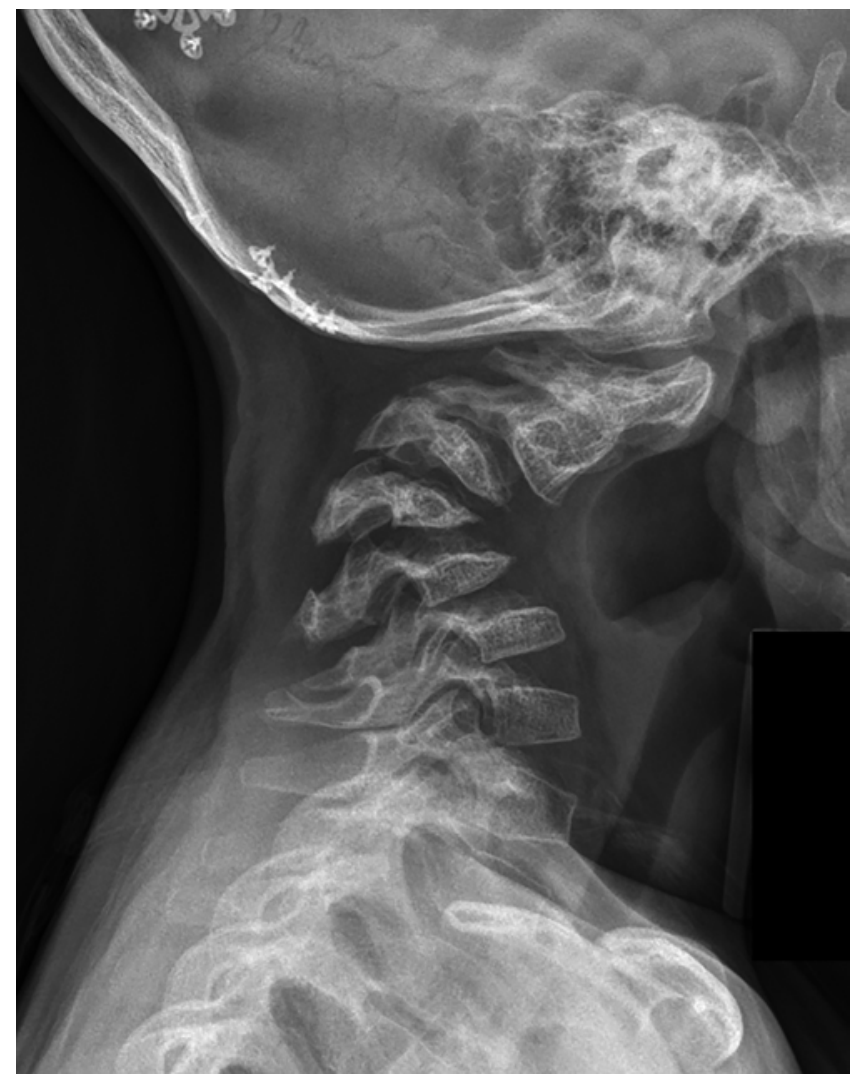

FIG. 1. Case 1. Lateral cervical spine radiograph of a 5.5-year-old girl who presented with postradiotherapy/laminectomy kyphosis of $88^{\circ}$ after having undergone a cervical spine ependymoma resection 4 years previously. Preoperative HGT was indicated due to progressive SCC and increased signal intensities seen on MRI. A halo crown with 8 pins torqued to 4-inch pounds was placed, and the duration of traction was set at 4 weeks.

and $3(43 \%)$ of 7 children with ARS that was successfully reduced in traction were treated with a halo vest as their definitive treatment. Posterior-only and combined anterior-posterior procedures were performed in 17 cases (68\%) and 8 cases (32\%), respectively. All 8 circumferential fusions were performed in cases of kyphosis. Nine occipitocervical (36\%), 3 atlantoaxial (12\%), 12 subaxial (48\%), and 1 occipitocervicothoracic (4\%) fusion were conducted. Surgical details were as follows: levels fused (median 3 levels [IQR 2-7]), estimated blood loss (median $200 \mathrm{ml}$ [IQR 88-575 ml]), anesthesia duration (median 343 minutes [IQR 249-450 minutes]), and surgical duration (median 276 minutes [IQR 180-384 minutes]). Three patients $(12 \%)$ experienced a decrease in IONM signals (2 with kyphosis and 1 with ARS), with 1 of the children developing a new neurological deficit that resulted in quadriplegia. This patient died at an outside institution after developing a complication at the tracheostomy site that resulted in an aortic-tracheal fistula that hemorrhaged.

The mean hospital length of stay (LOS) was 35 days (IQR 17-43 days). Of the 25 surgical patients, 18 children $(72 \%)$ were discharged in a halo vest and 7 (28\%) received rigid cervical collars. The mean follow-up period was 1.7 \pm 1.51 years (IQR $0.4-3$ years). In all 15 patients (100\%)

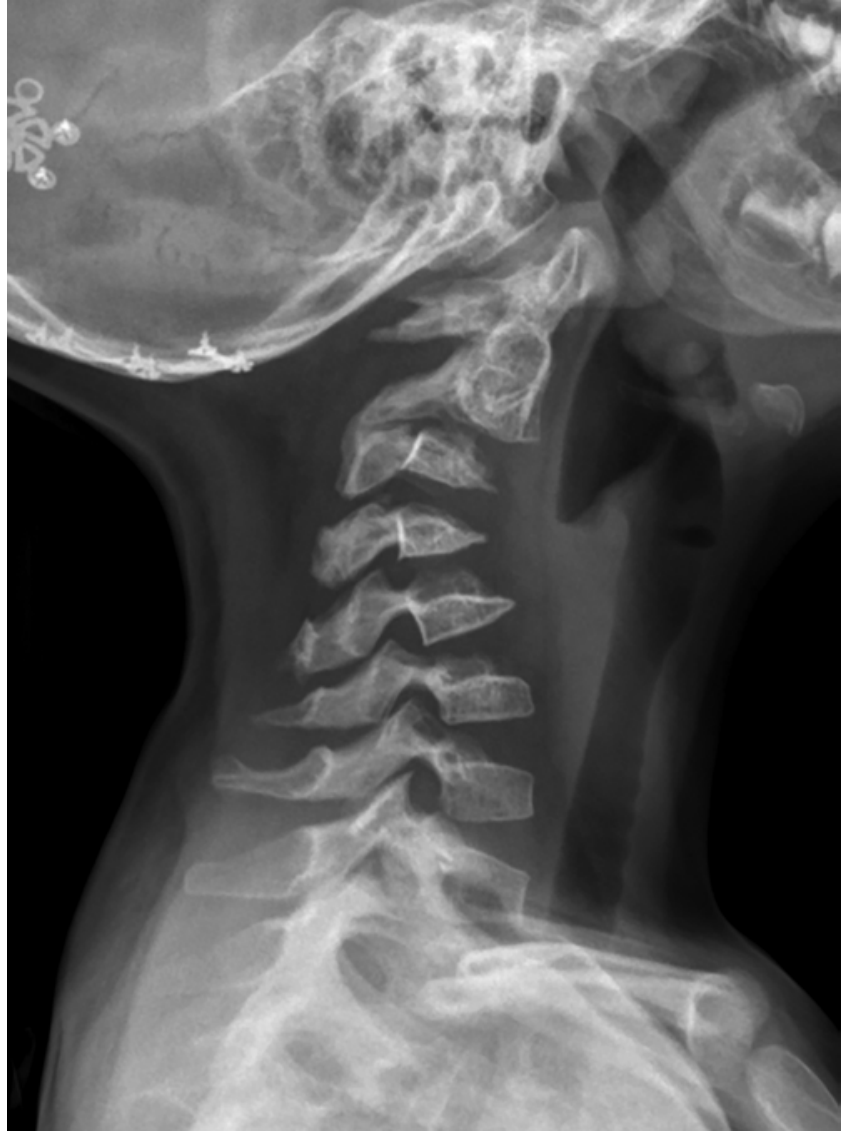

FIG. 2. Case 1. Lateral in-traction cervical spine radiograph after 3 weeks of HGT with 12 pounds $(5.4 \mathrm{~kg})$, or $34 \%$ of BW. The kyphosis has improved to $44^{\circ}$ ( $50 \%$ correction).

with sufficient follow-up, clinical and radiological fusion was present at the latest follow-up examination.

\section{Discussion}

Experience with HGT in the pediatric population with cervical spine issues is limited, with no reports in which safety or efficacy is evaluated.,10,13,14,16,18,24,28 Progressive cervical traction relaxes and elongates the soft tissues to gradually correct deformities in a controlled manner, alleviate neurological symptoms, assess deformity flexibility, decrease the force required during intraoperative reduction, minimize the risk of spinal cord injury, and increase correctability. ${ }^{14,16,22,29,30}$

Indications for HGT are difficult to quantify due to patient heterogeneity and the severity of pediatric cervical spine disorders, but conditions described in the literature include ARS, basilar invagination, tubercular spondylitis, os odontoideum, and kyphosis., ${ }^{1,13,14,16-18,24,29,31}$ Although not a standard of care, HGT is another tool that should be used in high-risk cases in which the surgeon believes achieving intraoperative reduction without traction is going to be significantly difficult.

\section{Cervical Kyphosis}

Some authors consider moderate-severe cervical ky- 


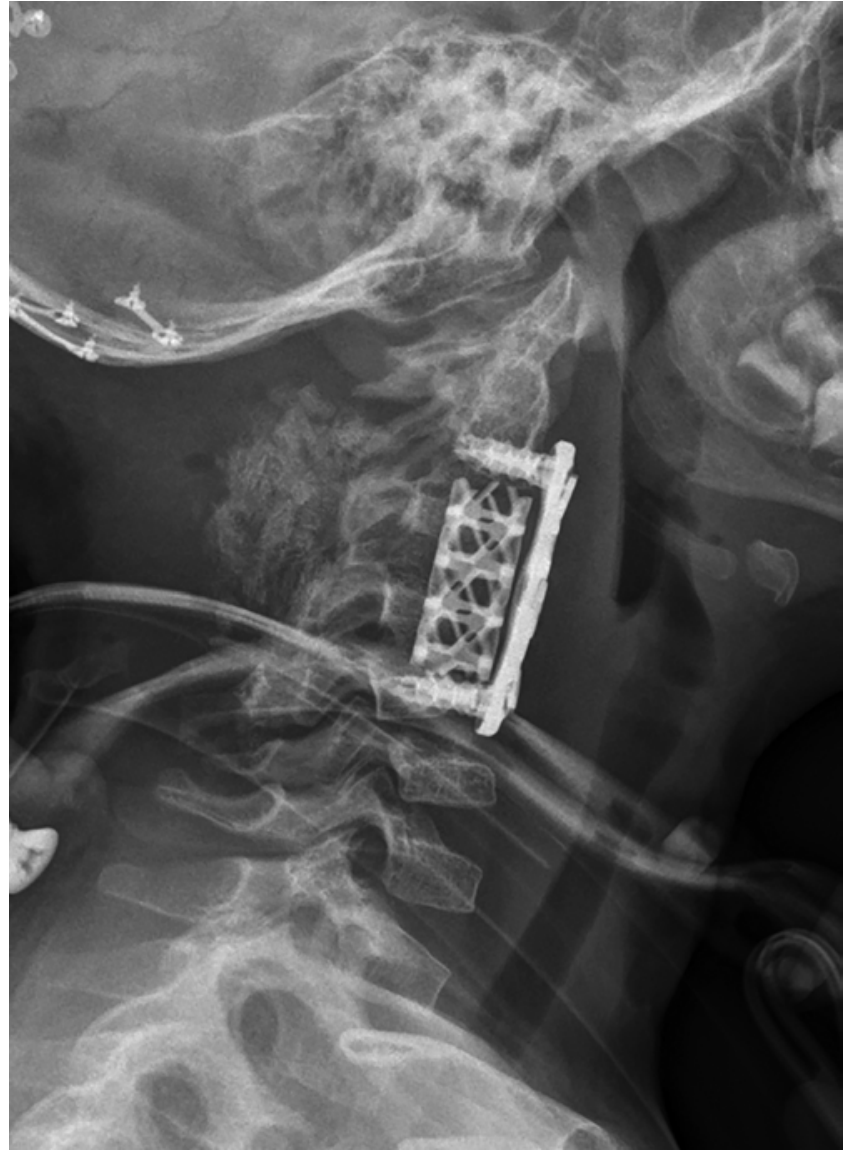

FIG. 3. Case 1. Postoperative lateral cervical spine radiograph showing further improvement of the kyphosis to $30^{\circ}$ after the patient underwent a combined anterior-posterior C2-5 fusion with anterior intervertebral cage placement. The posterior arthrodesis consisted of autograft combined with recombinant human bone morphogenetic protein. The patient awoke neurologically intact and was placed in a halo vest for 8 weeks. She was doing well clinically, and a solid posterior fusion mass was seen on radiographs at latest follow-up 8 months later.

phosis a contraindication for HGT use in children., ${ }^{5,25,31}$ Twelve patients in our series had cervical kyphosis $>60^{\circ}$, with a mean correction rate of $38 \%$ or $35^{\circ}$. This suggests that HGT is effective for treating cervical kyphosis in children and complements other studies that report improvement between $22^{\circ}$ and $85^{\circ} . .^{14,16}$

Kawabata et al. described 3 patients with neurofibromatosis type 1 (NF1) and severe kyphosis successfully treated with preoperative HGT. ${ }^{14}$ The mean pre-HGT and intraction kyphotic angles in those patients were $98^{\circ}$ (range $72^{\circ}-140^{\circ}$ ) and $52^{\circ}\left(\right.$ range $\left.50^{\circ}-55^{\circ}\right)$, respectively. However, the authors recommended less weight in patients with NF1 due to dystrophic bone and cervical spine fragility. Laohacharoensombat et al. also used less weight to treat an 8 -year-old boy with NF1 and severe kyphosis. ${ }^{16}$ After 12 days of traction with $1 \mathrm{~kg}$, a correction from $120^{\circ}$ to $90^{\circ}$ was seen in addition to improved neurological function. In a recent case series of 33 adults, Shen et al. used $9 \mathrm{~kg}$ in all patients regardless of body size and reported a mean kyphosis correction rate of $54.8 \% .{ }^{24}$ In that series, the duration of HGT was short (only 5-9 days).

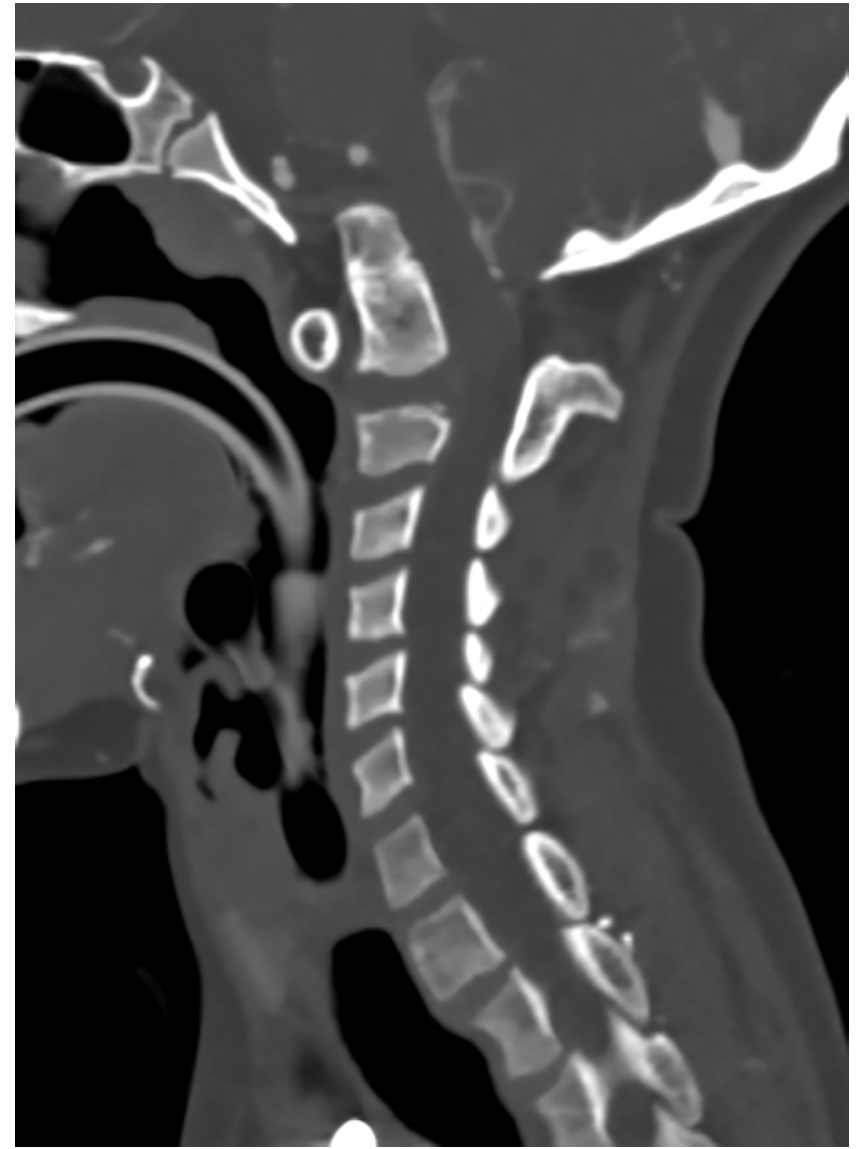

FIG. 4. Case 2. Sagittal cervical CT scan demonstrating severe basilar invagination in a 7.5-year-old boy with an uncharacterized chromosomal syndromic disorder who presented with myelopathy. The tip of the odontoid was $7.1 \mathrm{~mm}$ above McRae's line. The goal traction weight was set at 20 pounds $(8.16 \mathrm{~kg})$, or $28 \%$ of $\mathrm{BW}$, for 20 days.

The apex in cervical kyphosis experiences extreme compressive forces during surgical correction and predisposes the spinal cord to injury. ${ }^{5,14}$ The partial reduction achieved by HGT decreases the force required to achieve correction, diminishes the difficulty of anterior surgery in severely kyphotic patients, and can minimize the exposure area. ${ }^{14}$ The effects of HGT and surgery are additive, and we believe superior correction can be achieved using both as opposed to surgical intervention alone. ${ }^{24}$ Although attempts can be made with combined anterior-posterior procedures to improve correctability, increased complications and perioperative neurological deficits have been reported. ${ }^{13}$

\section{Basilar Invagination}

Cervical traction followed by posterior arthrodesis is less technically challenging than a combined single-stage procedure, reduces the likelihood of requiring a more invasive transoral dens resection, and improves the chances of a successful posterior occipitocervical fusion. ${ }^{8,11,17}$ Traction pulls the odontoid process below the foramen magnum under McRae's line to partially or completely reduce the invagination, alleviate brainstem compression, increase SAC, and improve neurological function. . $^{8,12,17,20,25}$ 


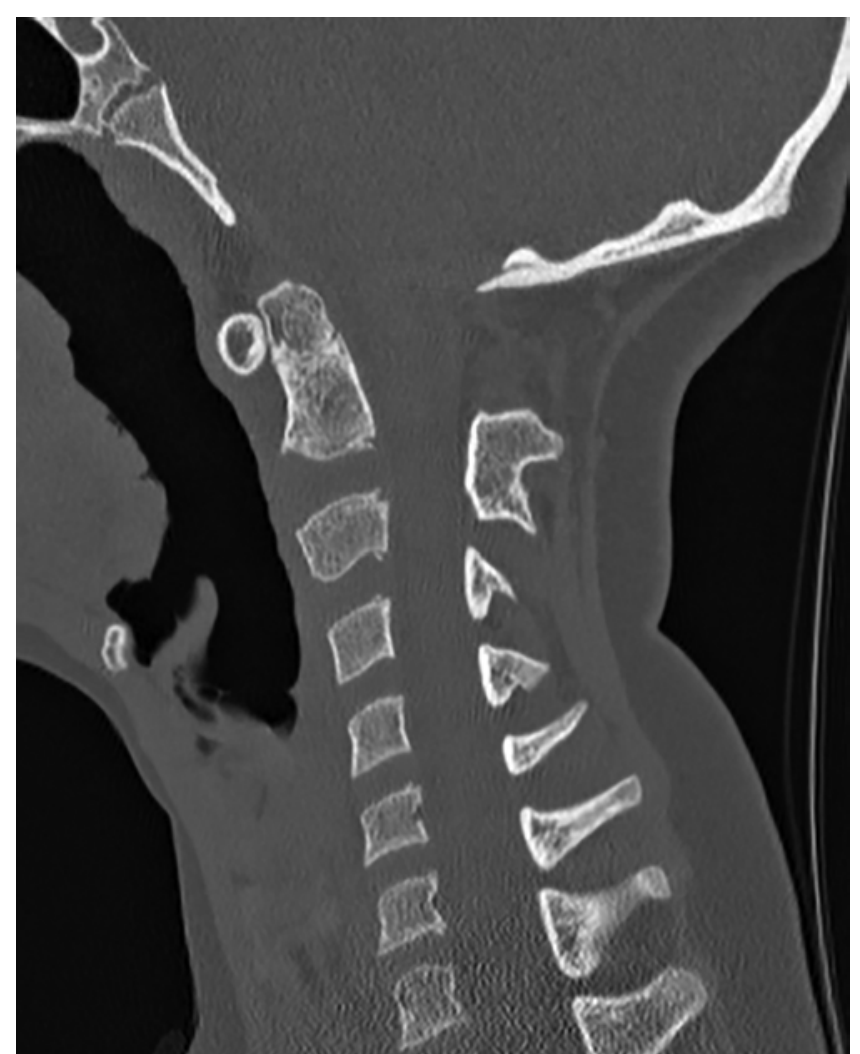

FIG. 5. Case 2. Sagittal in-traction CT scan after 2 weeks of HGT demonstrating a $12.9-\mathrm{mm}$ depression of the odontoid process, which is now located $5.9 \mathrm{~mm}$ below McRae's line and in correct anatomical orientation. Improvement of the atlantodental interval is also seen.

Intraoperative surgical screw and rod distraction maneuvers can further depress the odontoid to achieve the desired reduction.

Peng et al. reported good outcomes in 7 patients (age range 12-40 years) treated with Gardner-Wells tong traction for a mean of 21 days. ${ }^{11,17,20,23}$ All patients underwent posterior occipitocervical spinal fusion and avoided anterior surgery. HGT improves dens alignment but without the constraints of long-term immobilization required with Gardner-Wells traction. ${ }^{1,20}$ We found similar results for cases of displaced os odontoideum causing brainstem compression. Both patients were successfully treated with posterior occipitocervical arthrodesis and avoided anterior surgery. Li et al. also advocated preoperative HVGT in adults with irreducible ARS and basilar invagination to increase the chance of successful posterior-only decompression and arthrodesis. ${ }^{17}$

\section{Atlantoaxial Rotatory Subluxation}

HGT is not the first-line treatment for ARS. A trial of observation, cervical collar immobilization, or head halter traction should be attempted first, but irreducible and chronic cases require closed reduction with HGT with or without surgical stabilization. ${ }^{2,20}$ The goal of HGT differs from that of other cervical spine disorders in that nonoperative therapy may be adequate after successful closed reduction in traction. ${ }^{11}$ In this series, 3 (43\%) of 7 patients with ARS had successful reduction with traction and were treated with a halo vest as their definitive treatment.

\section{Complications}

All HGT-related AEs in this series were classified as minor grade I or II complications, suggesting that HGT is safe in the pediatric cervical spine population. Grade I complications, such as halo loosening and superficial pin-site infections, are the most frequent HGT-related AEs reported in the literature, with a prevalence of $16 \% .^{22,30}$ Complementary to current data, 4 superficial pin-site infections (14\%) were seen. These grade I complications are common intrinsic consequences of long-term invasive therapies, but they are easily treated with aggressive pinsite care or antibiotics. Other common grade I classifications are moderate to severe discomfort or pain that superimposes on the preexisting disorder and requires weight reduction or analgesics. ${ }^{30}$ Six children in the current series experienced transient neck discomfort or pain, but each was a sequela of the baseline condition and did not require weight removal or a change in management.

Moderate to severe paresthesias, cranial nerve dysfunction, or other nerve root injuries requiring a change in HGT protocol are classified as grade II complications. Damage to adjacent neurovascular structures can occur when exposed to stretching forces, and hypoglossal or other cranial nerve injuries are reported. ${ }^{10}$ Stretch neuropathies can be anticipated with daily neurological examinations, including a complete cranial nerve assessment and extremity sensorimotor evaluation.

We did not encounter any cranial nerve dysfunctions, but 4 transient sensory disturbances (14\%), classified as grade I complications, occurred but resolved spontaneously with clinical observation within 24-48 hours. Paresthesias occurred in $25 \%$ of cervical kyphosis cases, and this incidence is similar to that seen in other case series. ${ }^{30}$ Sensory disturbances occurred when greater weights were applied, and all resolved spontaneously. Although these AEs were common and important to recognize, grade I complications are minor events with little clinical relevance, did not cause a deviation from traction protocol, did not result in increased LOS or reoperations, and required minimal intervention. Most surgeons would agree that treatment of a pin-tract complication with oral antibiotics, while listed as a complication, is minimal with respect to the disease state being treated.

One patient developed a grade II complication. This patient presented with a preexisting neurological deficit and subsequently developed transient left-sided flaccid paralysis during HVGT initiation. Despite increased biomechanical stabilization with HVGT, traction likely aggravated and increased the SCC. The patient's motor strength returned after a 24-hour weight-free period, and no recurrence or additional neurological symptoms occurred after the rate of HVGT progression was reduced to 2 pounds $(0.91 \mathrm{~kg})$ per day. A possible explanation is that gradual soft-tissue relaxation improved the spinal alignment so that traction did not compromise the SAC. Bouchoucha et al. also described a case of neurological deterioration in a child with a preexisting deficit. ${ }^{4}$ The senior author (D.J.H.) believes that increased caution, slower weight progression, 


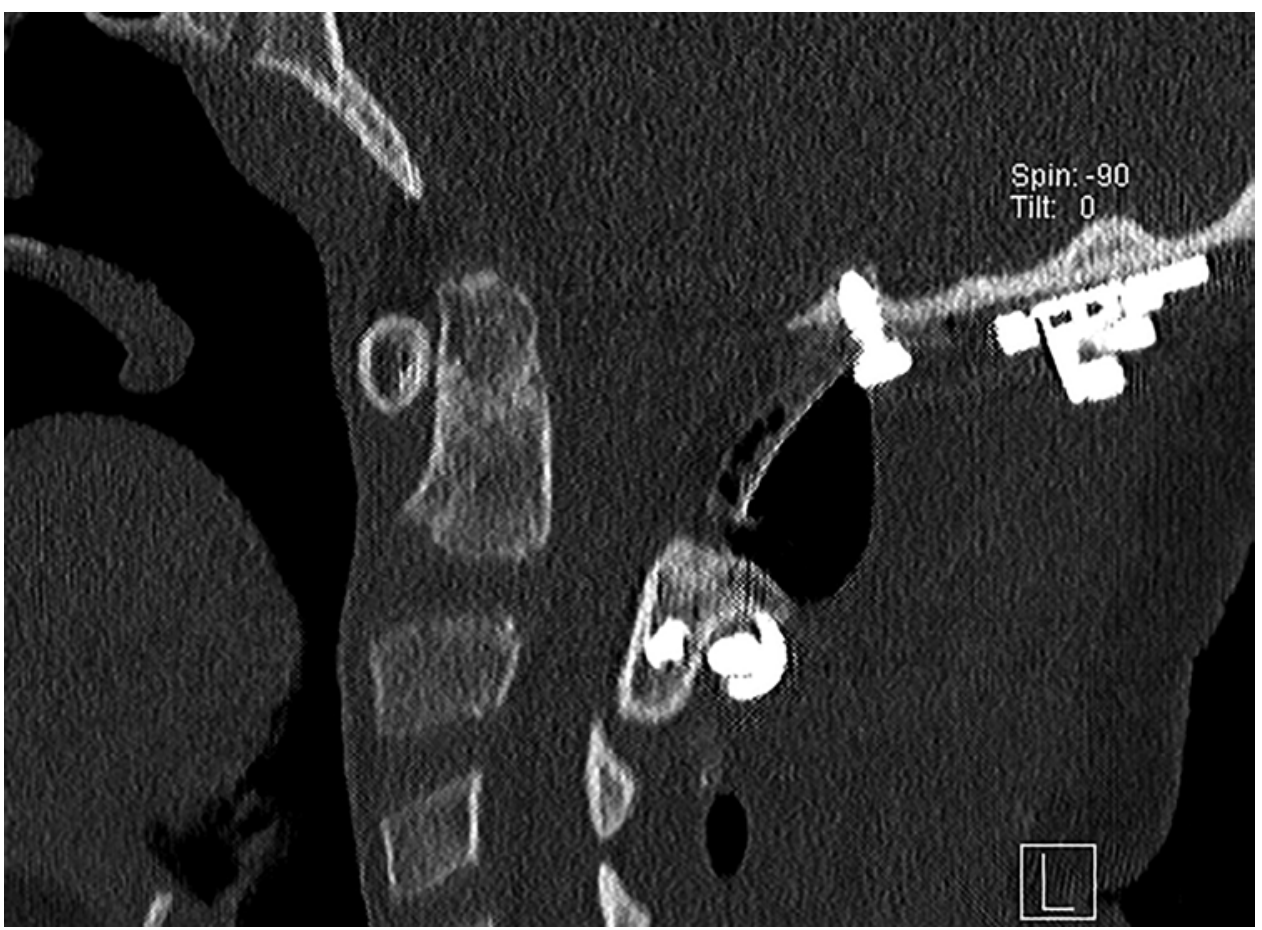

FIG. 6. Case 2. Postoperative sagittal CT scan after occipitocervical fusion (occiput-C3) demonstrating adequate reduction of the odontoid process below the foramen magnum. Preoperatively, the decision was made not to attempt $\mathrm{C} 1$ or $\mathrm{C} 2$ screw placement due to aberrant vertebral artery and vertebral body anatomy. The final construct consisted of an occipital plate, bilateral C3 translaminar screws, and bone grafting with iliac crest autograft, allograft, and recombinant human bone morphogenetic protein. The patient was immobilized in a halo vest for 3 months.

and HVGT are warranted in patients with preexisting neurological deficits or severe SCC to prevent catastrophic movement.

We acknowledge the limitations HGT may pose regarding LOS, healthcare expenditure, and family inconvenience. The senior author believes that these are acceptable given the significance of the problems in these children. It is difficult to determine the exact indications of HGT versus one-stage surgical correction without traction. We would agree that the indications are not well defined and much is based on the experience of the two senior authors and the large cohort they have had over the last 20 years. HGT is an additional tool in the arsenal of pediatric spine surgeons who manage severe cervical deformities. Cervical traction is an option if uncertainties exist that the spinal alignment required for internal fixation and deformity correction can be safely achieved in the operating room.

\section{Strengths and Limitations}

The retrospective design is the largest limitation. However, although we present the largest case series of HGT from an experienced pediatric tertiary referral center, the sample size remains small and may lack generalizability. Classification into various etiologies eliminated the potential for further statistical analysis, but this allowed for an interesting descriptive comparison with new insights that should be used as the basis for future prospective studies and meta-analyses.

One-year follow-up was not possible in some cases due to our international patient population and is a final limitation. The goal was to describe our experience with perioperative HGT. All patients had complete in-hospital records of their HGT admission, which was the objective of the study, and future prospective studies should analyze long-term outcomes or cost-effectiveness. To our knowledge, this is the first study to classify HGT complications using an objective complication classification system and is also the largest study to report on the feasibility, safety, and efficacy of HGT for the treatment of pediatric cervical spine disorders.

\section{Conclusions}

Preoperative HGT in children is safe and effective for gradual correction of severe cases of cervical kyphosis, atlantoaxial subluxation, basilar invagination, and os odontoideum. It provides the pediatric spine surgeon with another tool to manage extreme cases in which single-stage surgical reduction is high-risk or not feasible without partial alignment preoperatively. Grade I complications, such as superficial pin-site infections and transient paresthesias, occurred in $28 \%$ of our patients. Grade I complications are minor events with little clinical relevance, do not deviate from traction protocol, do not result in increased LOS or reoperation, and require minimal intervention. Traction with a HVGT may be warranted in patients with baseline neurological deficits and severe occipitocervical instability to reduce the chance of catastrophic movement that can occur with bending motions. 


\section{References}

1. Abd-El-Barr MM, Snyder BD, Emans JB, Proctor MR, Hedequist D: Combined preoperative traction with instrumented posterior occipitocervical fusion for severe ventral brainstem compression secondary to displaced os odontoideum: technical report of 2 cases. J Neurosurg Pediatr 25:724-729, 2016

2. Beier AD, Vachhrajani S, Bayerl SH, Aguilar CY, LambertiPasculli M, Drake JM: Rotatory subluxation: experience from the Hospital for Sick Children. J Neurosurg Pediatr 9:144-148, 2012

3. Bogunovic L, Lenke LG, Bridwell KH, Luhmann SJ: Preoperative halo-gravity traction for severe pediatric spinal deformity: complications, radiographic correction and changes in pulmonary function. Spine Deform 1:33-39, 2013

4. Bouchoucha S, Khelifi A, Saied W, Ammar C, Nessib MN, Ben Ghachem M: Progressive correction of severe spinal deformities with halo-gravity traction. Acta Orthop Belg 77:529-534, 2011

5. Crawford AH, Herrera-Soto J: Scoliosis associated with neurofibromatosis. Orthop Clin North Am 38:553-562, vii, 2007

6. Cronin CG, Lohan DG, Mhuircheartigh JN, Meehan CP, Murphy J, Roche C: CT evaluation of Chamberlain's, McGregor's, and McRae's skull-base lines. Clin Radiol 64:64-69, 2009

7. Dodwell ER, Pathy R, Widmann RF, Green DW, Scher DM, Blanco JS, et al: Reliability of the modified Clavien-DindoSink complication classification system in pediatric orthopaedic surgery. JBJS Open Access 3:e0020, 2018

8. Donnally CJ III, Munakomi S, Varacallo M (eds): Basilar Invagination. Treasure Island, FL: StatPearls Publishing, 2019

9. Emans J: Preliminary halo-gravity traction facilitates insertion of growing rods or VEPTR devices in severe early onset spinal deformity, presented at the 42nd Annual Meeting of the Scoliosis Research Society, September 5-8, 2007, Edinburgh, United Kingdom

10. Ginsburg GM, Bassett GS: Hypoglossal nerve injury caused by halo-suspension traction. A case report. Spine (Phila Pa 1976) 23:1490-1493, 1998

11. Goel A: Torticollis and rotatory atlantoaxial dislocation: a clinical review. J Craniovertebr Junction Spine 10:77-87, 2019

12. Goel A, Bhatjiwale M, Desai K: Basilar invagination: a study based on 190 surgically treated patients. J Neurosurg 88:962-968, 1998

13. Iwasaki M, Yamamoto T, Miyauchi A, Amano K, Yonenobu K: Cervical kyphosis: predictive factors for progression of kyphosis and myelopathy. Spine (Phila Pa 1976) 27:14191425,2002

14. Kawabata S, Watanabe K, Hosogane N, Ishii K, Nakamura M, Toyama Y, et al: Surgical correction of severe cervical kyphosis in patients with neurofibromatosis Type 1. J Neurosurg Spine 18:274-279, 2013

15. Koller H, Zenner J, Gajic V, Meier O, Ferraris L, Hitzl W: The impact of halo-gravity traction on curve rigidity and pulmonary function in the treatment of severe and rigid scoliosis and kyphoscoliosis: a clinical study and narrative review of the literature. Eur Spine J 21:514-529, 2012

16. Laohacharoensombat W, Wajanavisit W, Woratanarat P: Cervical neurofibromatosis with quadriparesis: management by fibular strut graft. Indian J Orthop 44:95-97, 2010

17. Li P, Bao D, Cheng H, Meng F, Li J: Progressive halo-vest traction preceding posterior occipitocervical instrumented fusion for irreducible atlantoaxial dislocation and basilar invagination. Clin Neurol Neurosurg 162:41-46, 2017

18. Mubarak SJ, Camp JF, Vuletich W, Wenger DR, Garfin SR: Halo application in the infant. J Pediatr Orthop 9:612-614, 1989

19. Nemani VM, Kim HJ, Bjerke-Kroll BT, Yagi M, Sacramento-Dominguez $\mathrm{C}$, Akoto $\mathrm{H}$, et al: Preoperative halo-gravity traction for severe spinal deformities at an SRS-GOP site in West Africa: protocols, complications, and results. Spine (Phila Pa 1976) 40:153-161, 2015

20. Peng X, Chen L, Wan Y, Zou X: Treatment of primary basilar invagination by cervical traction and posterior instrumented reduction together with occipitocervical fusion. Spine (Phila Pa 1976) 36:1528-1531, 2011

21. Powell EC, Leonard JR, Olsen CS, Jaffe DM, Anders J, Leonard JC: Atlantoaxial rotatory subluxation in children. Pediatr Emerg Care 33:86-91, 2017

22. Rinella A, Lenke L, Whitaker C, Kim Y, Park SS, Peelle $\mathrm{M}$, et al: Perioperative halo-gravity traction in the treatment of severe scoliosis and kyphosis. Spine (Phila Pa 1976) 30:475-482, 2005

23. Roye BD, Campbell ML, Matsumoto H, Pahys JM, Welborn $\mathrm{MC}$, Sawyer J, et al: Establishing consensus on the best practice guidelines for use of halo gravity traction for pediatric spinal deformity. J Pediatr Orthop 40:e42-e48, 2020

24. Shen X, Wu H, Shi C, Liu Y, Tian Y, Wu X, et al: Preoperative and intraoperative skull traction combined with anterior-only cervical operation in the treatment of severe cervical kyphosis (>50 degrees). World Neurosurg 130:e915-e925, 2019

25. Simsek S, Yigitkanli K, Belen D, Bavbek M: Halo traction in basilar invagination: technical case report. Surg Neurol 66:311-314, 2006

26. Sponseller PD, Takenaga RK, Newton P, Boachie O, Flynn J, Letko L, et al: The use of traction in the treatment of severe spinal deformity. Spine (Phila Pa 1976) 33:2305-2309, 2008

27. Stagnara P: [Cranial traction using the "Halo" of Rancho Los Amigos.] Rev Chir Orthop Reparatrice Appar Mot 57:287-300, 1971 (French)

28. Sucato DJ: Management of severe spinal deformity: scoliosis and kyphosis. Spine (Phila Pa 1976) 35:2186-2192, 2010

29. Taddonio RF Jr, King AG: Atlantoaxial rotatory fixation after decompressive laminectomy. A case report. Spine (Phila Pa 1976) 7:540-544, 1982

30. Yamamuro Y, Demura S, Murakami H, Kato S, Yonezawa N, Yokogawa N, et al: Acute progressive adolescent idiopathic cervical kyphosis: case report. J Neurosurg Spine 30:783787, 2019

31. Yang C, Wang H, Zheng Z, Zhang Z, Wang J, Liu H, et al: Halo-gravity traction in the treatment of severe spinal deformity: a systematic review and meta-analysis. Eur Spine J 26:1810-1816, 2017

32. Zeng H, Liang Y, Wang X, Shen X, Luo C, Xu Z, et al: Halo traction, single-segment circumferential fixation treating cervical tubercular spondylitis with kyphosis. Clin Neurol Neurosurg 138:59-65, 2015

\section{Disclosures}

The authors report no conflict of interest concerning the materials or methods used in this study or the findings specified in this paper.

\section{Author Contributions}

Conception and design: all authors. Acquisition of data: Verhofste, O'Neill. Analysis and interpretation of data: all authors. Drafting the article: all authors. Critically revising the article: all authors. Reviewed submitted version of manuscript: all authors. Approved the final version of the manuscript on behalf of all authors: Hedequist. Statistical analysis: Verhofste. Study supervision: Hedequist.

\section{Correspondence}

Daniel J. Hedequist: Boston Children's Hospital, Boston, MA. daniel.hedequist@childrens.harvard.edu. 